Qin-Juan Xu*

\title{
Crystal structure of catena-poly[bis $\left(\mu_{2^{-}}\right.$ thiocyanato- $\left.\mathrm{K}^{2} N: S\right)$-(2-(5-methyl-1H-pyrazol-3-yl) pyridine- $\left.{ }^{2} N, N^{\prime}\right)$ cadmium(II)]-dioxane (1/1), $\mathrm{C}_{15} \mathrm{H}_{17} \mathrm{CdN}_{5} \mathrm{O}_{2} \mathrm{~S}_{2}$
}

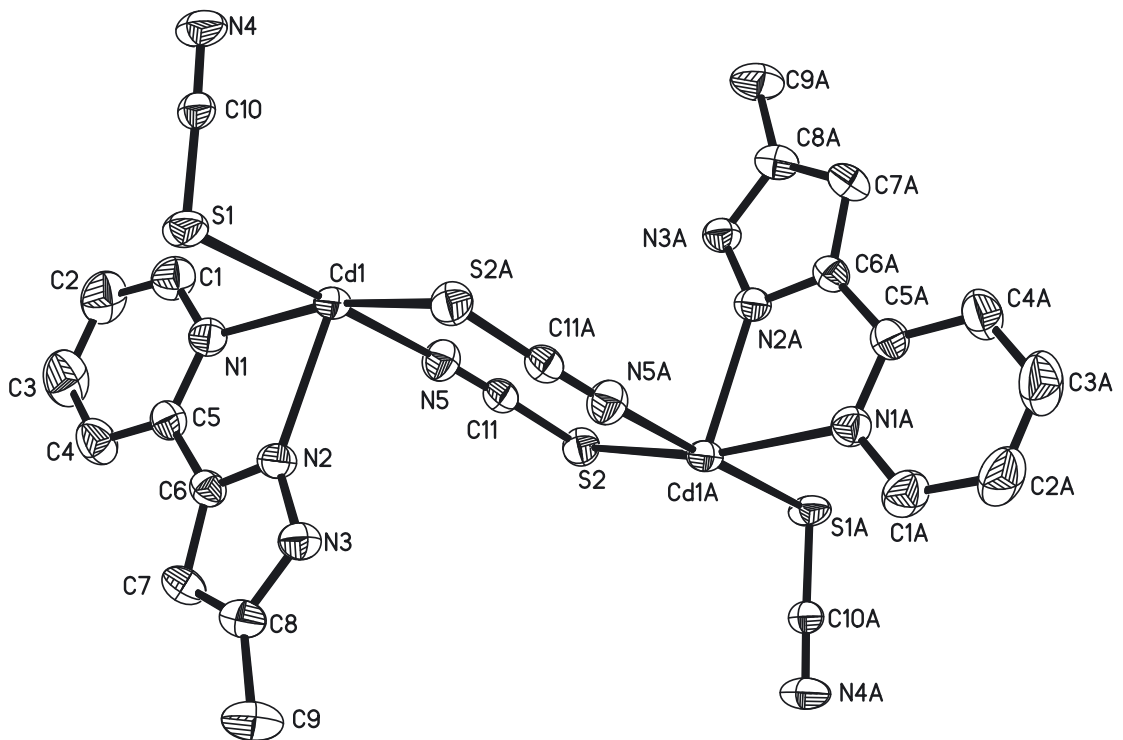

https://doi.org/10.1515/ncrs-2021-0019

Received January 13, 2021; accepted February 18, 2021;

published online March 11, 2021

\section{Abstract}

$\mathrm{C}_{15} \mathrm{H}_{17} \mathrm{CdN}_{5} \mathrm{O}_{2} \mathrm{~S}_{2}$, triclinic, $P \overline{1}$ (no. 2), $a=9.6685(4) \AA$, $b=10.4511(4) \AA, c=11.2840(5) \AA, \alpha=100.763(2)^{\circ}$, $\beta=114.948(2)^{\circ}, \gamma=96.816(2)^{\circ}, V=990.36(7) \AA^{3}, Z=2$, $R_{\mathrm{gt}}(F)=0.0316, w R_{\mathrm{ref}}\left(F^{2}\right)=0.1169, T=296(2) \mathrm{K}$.

\section{CCDC no.: 2063620}

Table 1 contains crystallographic data and Table 2 contains the list of the atoms including atomic coordinates and displacement parameters.

*Corresponding author: Qin-Juan Xu, Weihai Ocean Vocational College, Rongcheng, Shandong 264300, P. R. China,

E-mail: 15662370667@163.com. https://orcid.org/0000-0002-2910209X
Table 1: Data collection and handling.

\begin{tabular}{ll}
\hline Crystal: & Colorless block \\
Size: & $0.10 \times 0.05 \times 0.05 \mathrm{~mm}$ \\
Wavelength: & Mo $K \alpha$ radiation $(0.71073 \AA)$ \\
$\mu:$ & $1.33 \mathrm{~mm}^{-1}$ \\
Diffractometer, scan mode: & Bruker SMART APEX II, $\varphi$ and $\omega$ \\
$\theta_{\text {max }}$, completeness: & $27.8^{\circ},>99 \%$ \\
$N(h k l)_{\text {measured }}, N(h k l)_{\text {unique }}, R_{\text {int }}:$ & $16640,4605,0.028$ \\
Criterion for $I_{\text {obss }}, N(h k l)_{\text {gt }}:$ & $I_{\text {obs }}>2 \sigma\left(I_{\text {obs }}\right), 3901$ \\
$N(\text { param })_{\text {refined }}:$ & 226 \\
Programs: & Bruker [1], SHELX [2, 3], Diamond [4]
\end{tabular}

\section{Source of material}

First, 3-(4-pyridyl)pyrazole was prepared by using 4-acetyl pyridine, $\mathrm{N}, \mathrm{N}$-dimethylacetamide dimethyl acetal (CAS:18871-66-4) and hydrazine under certain conditions according to the described method $[5,6]$. Then, the $1 \mathrm{~mL}$ aqueous solution of $\mathrm{CdCl}_{2}(0.1 \mathrm{~mol} / \mathrm{L})$ and $\mathrm{KSCN}(0.2 \mathrm{~mol} / \mathrm{L})$ was slowly dropped into the $10 \mathrm{~mL}$ acetonitrile solution of 2-(5-methyl-1H-pyrazol-3-yl)pyridine $(15.9 \mathrm{mg}, 0.1 \mathrm{mmol})$ 
Table 2: Fractional atomic coordinates and isotropic or equivalent isotropic displacement parameters $\left(\AA^{2}\right)$.

\begin{tabular}{|c|c|c|c|c|}
\hline Atom & $x$ & $y$ & $z$ & $U_{\text {iso }} * / U_{\text {eq }}$ \\
\hline d1 & $0.15298(2)$ & $0.28051(2)$ & $0.55214(2)$ & $0.04682(11)$ \\
\hline S1 & $0.26784(11)$ & $0.12944(9)$ & $0.73965(9)$ & $0.0574(2)$ \\
\hline 2 & $-0.03993(11)$ & $0.61617(10)$ & $0.28509(10)$ & 0.0561 (2) \\
\hline N1 & $0.3343(3)$ & $0.2180(3)$ & $0.4753(3)$ & $0.0541(7)$ \\
\hline $\mathrm{N} 2$ & $0.3921(3)$ & $0.4388(3)$ & $0.6743(3)$ & $0.0476(6)$ \\
\hline N3 & $0.4514(3)$ & $0.5544(3)$ & $0.7716(3)$ & $0.0509(6)$ \\
\hline 13 & 0.400700 & .590000 & 811101 & $0.061^{*}$ \\
\hline N4 & $0.0388(4)$ & $37(3)$ & $982(3)$ & $0.0707(10)$ \\
\hline N5 & $0.0613(4)$ & $0.4092(3)$ & $0.4000(3)$ & $0.0583(7)$ \\
\hline $\mathrm{C} 1$ & $0.3082(5)$ & $0.1052(4)$ & $0.3823(4)$ & $0.0704(11)$ \\
\hline H1 & 0.207900 & 0.049600 & .339600 & $0.085^{*}$ \\
\hline $\mathrm{C} 2$ & $0.4176(7)$ & $0.0676(5)$ & $0.3471(5)$ & $0.0906(15)$ \\
\hline $\mathrm{H} 2$ & 394100 & -0.012100 & .282700 & $0.109^{\star}$ \\
\hline C3 & $0.5607(7)$ & 7 (6) & $0.4079(6)$ & $0.0979(18)$ \\
\hline $3 A$ & 8801 & 901 & 5100 & $0.118^{\star}$ \\
\hline C4 & $0.5920(5)$ & $0.2649(5)$ & $0.5033(4)$ & $0.0732(11)$ \\
\hline H4 & 691001 & 901 & 6399 & $0.088^{*}$ \\
\hline $\mathrm{C} 5$ & $0.4772(4)$ & $1(4)$ & $2(3)$ & $0.0513(8)$ \\
\hline C6 & $5(4)$ & (3) & $6(3)$ & $0.0456(7)$ \\
\hline $\mathrm{C} 7$ & $(4)$ & (4) & $0(4)$ & $4(8)$ \\
\hline $\mathrm{H} 7$ & 730900 & 100 & 201 & $0.068^{\star}$ \\
\hline $\mathrm{C} 8$ & $5(4)$ & $4(4)$ & (4) & $68(8)$ \\
\hline C9 & $0.6895(6)$ & $0.7345(5)$ & $0.9058(5)$ & $0.0841(13)$ \\
\hline H9A & 626600 & 600 & 945499 & $.127^{\star}$ \\
\hline $9 B$ & 0.720100 & 800 & .865801 & $.127^{\star}$ \\
\hline$-19 C$ & 781700 & & 399 & $.127^{\star}$ \\
\hline C10 & $318(4)$ & $-0.0065(3)$ & $2(3)$ & 0.0439 (7) \\
\hline C11 & $8(4)$ & $8(3)$ & $35(3)$ & $0.0460(7)$ \\
\hline 12 & $0.6930(6)$ & $0.3342(5)$ & $-0.0362(4)$ & $0.0774(12)$ \\
\hline $\mathrm{H} 12 \mathrm{~A}$ & 0.691801 & 0.428201 & -0.028700 & $0.094^{*}$ \\
\hline $\mathrm{H} 12 \mathrm{~B}$ & 400 & 399 & 9699 & $0.094^{\star}$ \\
\hline C13 & $0.8395(7)$ & $0.3095(6)$ & $91(5)$ & 0.0855 (14) \\
\hline $\mathrm{H} 13 \mathrm{~A}$ & 0.844300 & 0.331700 & -0.117200 & $0.103^{*}$ \\
\hline H13B & 929200 & 401 & .041600 & $0.103^{*}$ \\
\hline C14 & $0.8431(6)$ & $0.1414(5)$ & 0.0699 (5) & $0.0832(13)$ \\
\hline $\mathrm{H} 14 \mathrm{~A}$ & 0.932599 & 0.196600 & 0.153101 & $0.100^{*}$ \\
\hline $\mathrm{H} 14 \mathrm{~B}$ & 0.849000 & .049100 & 0.065500 & $0.100^{*}$ \\
\hline C15 & $0.6954(6)$ & $8(5)$ & $2(5)$ & 0.0799 (12) \\
\hline $\mathrm{H} 15 \mathrm{~A}$ & 0.608400 & 0.105301 & -0.008900 & $0.097^{\star}$ \\
\hline $15 B$ & 0.692201 & 142100 & 0.149900 & $0.097^{\star}$ \\
\hline 01 & $0.6821(4)$ & $0.2983(3)$ & $0.0757(3)$ & $0.0698(7)$ \\
\hline 02 & $0.8531(5)$ & $0.1753(4)$ & $-0.0434(3)$ & $0.0909(10)$ \\
\hline
\end{tabular}

and triethylamine ( $16 \mathrm{~mL}, 0.1 \mathrm{mmol})$. The mixture solution was stirred at room temperature for $30 \mathrm{~min}$. After filtration, it was stood to evaporate for one week to form colorless block crystals of the title compound.

\section{Experimental details}

$\mathrm{H}$ atoms were added geometrically. Their $U_{\text {iso }}$ values were set to $1.2 U_{\text {eq }}$ of the parent atoms.

\section{Comment}

2-(5-Methyl-1H-pyrazol-3-yl)pyridine have been widely studied as important ligands [7], which has the similar bidentate coordination sites as the magnetism-active ligands $[8,9]$. These ligands usually transmit antiferromagnetic coupling.

In the title compound, all the bond lengths are within normal ranges, similar to its mononuclear analogue $[5,10]$. The Cd is chelated by one 2-(5-methyl-pyrazolate-3-yl)pyridine, and bridged by two $\mathrm{SCN}^{-}$to form a $1 \mathrm{D}$ chain. The chains of the title compound are interlinked to form 3D supramolecular networks by one classical intermolecular H-bond N3-H3A $\cdots 01(-x+1,-y+1,-z+1)$ (length 2.890(2) $)$.

Author contributions: All the authors have accepted responsibility for the entire content of this submitted manuscript and approved submission.

Research funding: Shandong Province Inorganic and Analytical Chemistry Excellent Resource Sharing Course (2018) and Luan Hui-ni Famous Teacher Studio (2019).

Conflict of interest statement: The authors declare no conflicts of interest regarding this article.

\section{References}

1. Bruker. APEX2; Bruker AXS Inc.: Madison, USA, 2011.

2. Sheldrick G. M. SHELXTL - Integrated space-group and crystalstructure determination. Acta Crystallogr. 2015, A71, 3-8.

3. Sheldrick G. M. Crystal structure refinement with SHELXL. Acta Crystallogr. 2015, C71, 3-8.

4. Brandenburg K. DIAMOND. Visual Crystal Structure Information System. Ver. 4.0; Crystal Impact: Bonn, Germany, 2015.

5. Cai H., Guo Y., Li J.-G. Bis[2-(1H-pyrazol-3-yl-k $\left.N^{2}\right)$ pyridine-k $N$ ] dithiocyanato-kN,kS-cadmium(II). Acta Crystallogr. 2010, E66, m1205.

6. Yang F.-L., Zhu G.-Z., Liang B.-B., Shi Y.-H., Li X.-L. Assembly of dinuclear copper(II) complexes based on a tridentate pyrazolpyridine ligand: crystal structures and magnetic properties. Polyhedron 2017, 128, 104-111.

7. Wu W.-H., Wang Y.-Q., Mi H.-L., Xue Q.-X., Shao F., Shen F., Yang F.-L. Magnetic relaxation in a Co(II) chain complex: synthesis, structure, and DFT computational coupling constant. CrystEngComm 2021, 23, 1398-1405.

8. Yang F. L., Chen X., Wu W. H., Zhang J. H., Zhao X. M., Shi Y. H., Shen F. Spin switching in tris(8-aminoquinoline)iron(II) $\left(\mathrm{BPh}_{4}\right)_{2}$ : quantitative guest-losing dependent spin crossover properties and single-crystalto-single-crystal transformation. Dalton Trans. 2019, 48, 231-241.

9. Yang F.-L., Liang B.-B., Zhang J.-H., Wu W.-H., Zheng S.-Q., Mao Y.-Y., Chen J.-Q., Cao J.-X., Zhu G.-Z. Synthesis, structures and magnetic properties of three copper(II) complexes of 2-(1Hpyrazol-3-yl)pyridine. Transition Met. Chem. 2018, 43, 211-220.

10. Ling N., Wang X., Zhang Y.-W., Zhao T.-T., Ruan Y., Yang J. Crystal structure of dimethanol-bis(1-((2-methyl-1H-benzo[d]imidazol-1-yl) methyl)-1H-benzo[d] [1,2,3]triazole-kN)-bis(thiocyanato-kN) cadmium(II) C34H34CdN12O2S2. Z. Kristallogr. NCS 2019, 234, 881-882. 